\title{
High fructose corn syrup, excess-free- fructose, and risk of coronary heart disease among African Americans- the Jackson Heart Study
}

\author{
Luanne R. DeChristopher ${ }^{{ }^{*}}$ (D, Brandon J. Auerbach ${ }^{2}$ and Katherine L. Tucker $^{3}$
}

\begin{abstract}
Background: Researchers have sought to explain the black-white coronary heart disease (CHD) mortality disparity that increased from near parity to 30\% between 1980 and 2010. Contributing factors include cardiovascular disease prevention and treatment disparities attributable to disparities in insurance coverage. Recent research suggests that dietary/environmental factors may be contributors to the disparity. Unabsorbed/luminal fructose alters gut bacterial load, composition and diversity. There is evidence that such microbiome disruptions promote hypertension and atherosclerosis. The heart-gut axis may, in part, explain the black-white CHD disparity, as fructose malabsorption prevalence is higher among African Americans. Between 1980 and 2010, consumption of excess-free-fructose-the fructose type that triggers malabsorption-exceeded dosages associated with fructose malabsorption $(\sim 5 \mathrm{~g}-10 \mathrm{~g})$, as extrapolated from food availability data before subjective, retroactively-applied loss adjustments. This occurred due to an industrial preference shift from sucrose to high-fructose-corn-syrup (HFCS) that began 1980. During this period, HFCS became the main sweetener in US soda. Importantly, there has been more fructose in HFCS than thought, as the fructose-to-glucose ratio in popular sodas (1.9-to-1 and 1.5-to-1) has exceeded generally-recognized-as-safe levels (1.2-to-1). Most natural foods contain a 1-to-1 ratio. In one recent study, $\geq 5$ times/wk. consumers of HFCS sweetened soda/fruit drinks/and apple juice-high excess-free-fructose beverages-were more likely to have CHD, than seldom/never consumers.

Methods: Jackson-Heart-Study data of African Americans was used to test the hypothesis that regular relative to low/ infrequent intake of HFCS sweetened soda/fruit drinks increases CHD risk, but not orange juice-a low excess-free-fructose juice. Cox proportional hazards models were used to calculate hazard ratios using prospective data of 3407-3621 participants, aged 21-93 y (mean 55 y).

Results: African Americans who consumed HFCS sweetend soda 5-6x/wk. or any combination of HFCS sweetened soda and/or fruit drinks $\geq 3$ times/day had $\sim 2$ (HR 2.08, 95\% Cl 1.03-4.20, $P=0.041$ ) and 2.5-3 times higher CHD risk (HR 2.98, 95\% Cl 1.15-7.76; $P=0.025$ ), respectively, than never/seldom consumers, independent of confounders. There were no associations with diet-soda or 100\% orange-juice, which has a similar glycemic profile as HFCS sweetened soda, but contains a 1:1 fructose-to-glucose ratio.

(Continued on next page)
\end{abstract}

\footnotetext{
* Correspondence: luanne.dechristopher@gmail.com

'Independent Researcher, M.Sc. Biochemistry, Molecular Biology, P.O. Box

5542, Eugene, OR 97405, USA

Full list of author information is available at the end of the article
}

C C The Author(s). 2020 Open Access This article is licensed under a Creative Commons Attribution 4.0 International License, which permits use, sharing, adaptation, distribution and reproduction in any medium or format, as long as you give appropriate credit to the original author(s) and the source, provide a link to the Creative Commons licence, and indicate if changes were made. The images or other third party material in this article are included in the article's Creative Commons licence, unless indicated otherwise in a credit line to the material. If material is not included in the article's Creative Commons licence and your intended use is not permitted by statutory regulation or exceeds the permitted use, you will need to obtain permission directly from the copyright holder. To view a copy of this licence, visit http://creativecommons.org/licenses/by/4.0/. The Creative Commons Public Domain Dedication waiver (http://creativecommons.org/publicdomain/zero/1.0/) applies to the data made available in this article, unless otherwise stated in a credit line to the data. 
(Continued from previous page)

Conclusion: The ubiquitous presence of HFCS in the food supply may pre-dispose African Americans to increased CHD risk.

Keywords: African Americans, Health race disparities, Heart disease, High fructose corn syrup, Fructose malabsorption , Fructose, Fruit drinks, Glycation, Soda, Soft drinks, Microbiome, Dyspepsia, AGE, FruAGE, Excess-free-fructose, Fructositis

\section{Background}

Researchers have sought to explain the black-white coronary heart disease (CHD) mortality disparity that increased from near parity to $\sim 30 \%$ between 1980 and 2008 [1]. By 2010, African Americans were 30\% more likely to die from heart disease than non-Hispanic whites $[2,3]$. Disparities in cardiovascular disease prevention and treatment have been cited as contributing factors [4]. Non-Hispanic blacks are twice as likely to be uninsured, compared with non-Hispanic whites, in part because blacks have experienced a disproportionate decline in employer-sponsored health insurance coverage since the 1970s. Studies indicate that those who are uninsured go without needed medical care, receive lower quality care and have worse health, compared with those who have health insurance [5].

Dietary/environmental stressors are potential contributors to the black-white heart disease mortality disparity. Epidemiological research shows that regular consumers of sugar sweetened beverages (SSB) (high fructose corn syrup sweetened soda, and fruit drinks) have increased CHD risk, relative to seldom/never consumers, due to sugar metabolism mechanisms linked to SSB and, in particular, (unregulated) fructose overconsumption (hypertension, dyslipidemia induced atherosclerosis and inflammation) [6-8]. Research with nationally representative data found that $\mathrm{SSB} /$ fruit drink consumption prevalence was higher among African Americans than non-Hispanic whites [9].

A recent study using nationally representative data found that the increased CHD risk may, in part, be due to the the high fructose-to-glucose ratio in the high fructose corn syrup (HFCS) in soda and fruit drinks and mechanisms linked to fructose malabsorption. Consumers of any combination of HFCS sweetened soda, fruit drinks and apple juice - a 100\% juice with a high $(\sim 2: 1)$ fructose-to-glucose ratio $-\geq 5$ times/wk. were nearly three times as likely to have CHD, relative to seldom/never consumers [10]. There was no association with orange juice-a juice with comparable amounts of total sugars $(20.7 \mathrm{~g})$ [11], total fructose (11 g), glycemic load (15 glycemic units) [12], and post pasteurization vitamin $C$ as apple juice $(24 \mathrm{~g}) /(15.7 \mathrm{~g})$ [11]/(12 glycemic units) [12], per $250 \mathrm{ml}$ ( $8 \mathrm{oz}$ cup). What distinguishes these juices is their excess-free-fructose (unpaired fructose) content (7.4 g in apple juice (NDB \#09400) [11] vs. $0.4 \mathrm{~g}$ in orange juice, (NDB \#09209) [11] per $250 \mathrm{ml}$.)
Between 1980 and 2010, consumption of excess-freefructose exceeded dosages associated with fructose malabsorption $(\sim 5 \mathrm{~g}-10 \mathrm{~g})$ [13-22], as extrapolated from food availability data [23, 24] before subjective, retroactively applied loss adjustments [25-27]. Fructose malabsorption may be an underlying CHD risk factor, as unabsorbed/luminal fructose alters gut bacterial load, composition and diversity [28], and recent research shows that such detrimental shifts in the microbiome promote hypertension and atherosclerosis [29, 30]. African Americans may be at increased risk, as fructose malabsorption prevalence is higher among non-Hispanic blacks relative to Hispanics, despite the fact that both ethnicities exhibit high consumption of HFCS sweetened beverages [31]. HFCS has been the main source of excess-freefructose in the US food supply, due to the industrial preference shift from sucrose to HFCS [32, 33]. Excess-free- fructose occurs when the fructose-to-glucose ratio exceeds 1:1. Most natural foods (including honey) [34] contain a 1:1 ratio. Apples, pears, watermelons, and mangoes are among the exceptions [11]. Importantly, there has been more fructose in HFCS than previously thought, as research from the Keck School of Medicine found that the fructose-toglucose ratio in popular sodas (1.9 to 1 [35] and 1.5 to 1 [36]) has exceeded generally recognized as safe (GRAS) levels [37] (1.2 to 1). The proliferation of HFCS in the US food supply between 1980 and 2010 may have contributed to the black/white heart disease mortality disparity.

\section{Study objectives}

We sought to investigate whether African Americans, who consume high excess-free-fructose beverages, have higher CHD risk than seldom/never consumers. This study tests the hypothesis that consumption of HFCS sweetened soda, fruit drinks, and any combination of these high excess-free-fructose beverages, but not orange juice (a 100\% juice with a 1:1 fructose-to-glucose ratio [11]) or diet-soda, increases CHD risk, independently of potential confounders. Survival analysis was conducted with data from the Jackson Heart Study (JHS), a population-based longitudinal study of African Americans based in Jackson, Mississippi [38]. The JHS is the largest single-site, epidemiologic investigation of cardiovascular disease (CVD) among African Americans $(n=$ 5306) undertaken in the United States. It aims to elucidate the reasons for the greater prevalence of CVD among African Americans [38]. 
By 2000, the start of the baseline exam, participants in the JHS were exposed to higher intakes of excess-freefructose than generations before them, as US soft drink producers switched from use of sucrose to HFCS in non-diet soda around 1984 [24, 39]. Average per capita HFCS intake peaked the year (1999) before the JHS began, at approximately $80 \mathrm{~g}$ per day (over 1 pound per week) $[23,24]$. These consumption data are consistent with US Department of Agriculture reports [23] published prior to subjective increases in HFCS consumer level loss allowances [25]. The JHS was approved by the US-National Heart, Lung and Blood Institute of the National Institutes of Health. Participants gave written consent. The study was approved by the Institutional Review Board of the University of Mississippi [38].

\section{Methods}

\section{Beverage intake}

We analyzed intake of non-diet soda, the high excessfree-fructose beverage most consumed by US adults [9, 40] and fruit drinks, as many varieties are sweetened with HFCS and/or contain apple juice (a 100\% juice which naturally contains $\mathrm{a} \geq 2: 1$ fructose-to-glucose ratio [11]). These beverages were analyzed individually and in combination with one another, herein referred to as any combination of HFCS sweetened soda and fruit drinks (ttlEFF - total excess-free-fructose). Orange juice, a $100 \%$ juice with a 1:1 fructose-to-glucose ratio [11], and diet soda were included for comparison. Although we also analyzed other $100 \%$ fruit juices, asked as, "Do you consume other $100 \%$ juices (other than orange juice), including apple juice?" [38], this beverage group did not distinguish between non-citrus juice that is high in excess-free-fructose (apple) [11], and other 100\% juices with low/no excess-free-fructose (grape, grapefruit and pineapple) [11]. Grouping these juices into one question prevented individual analysis of apple juice, as distinguished from other popular juices [38]. During the study period, HFCS was the main sweetener in US soda [41].

For analysis, individual beverage intake frequencies, obtained via a food frequency questionnaire (FFQ) specifically designed for this population [42], were reduced from ten to four ( $\leq$ once/wk., 2-4 times/wk., 5-6 times/ wk., and $\geq$ once a day), except for individual analyses with diet soda, with three intake levels ( $\leq$ once/wk., 2-6 times/wk., zonce a day), as fewer people reported diet soda consumption. To analyze any combination of nondiet soda and fruit drinks, we categorized summed frequencies (normalized to $237 \mathrm{ml}$ or one $8 \mathrm{oz}$ cup) into quintiles. All intake frequencies were carried forward from exam 1 to exams 2 and 3, as the JHS administered one FFQ at enrollment. Strong and consistent relationships have been reported between frequency of food and food group consumption and probability of consumption on $24 \mathrm{~h}$ recalls [43] and, in a validation and calibration study, the FFQ used in the JHS, as described in-depth elsewhere, was found to be reasonably valid for assessment of dietary intake of adult African Americans in the south $[42,44]$.

\section{Ascertainment of endpoints}

Incident $\mathrm{CHD}$ was defined as any one of the following: self-reported non-fatal myocardial infarction (MI), as determined by 12-LED electrocardiogram; fatal MI as determined by review of hospital records and death certificates; and self-reported CHD, based on answers to the following questions: "Since your last JHS visit, have you ... had coronary bypass? ... had angioplasty of the coronary arteries? ... had a balloon angioplasty or stent of the coronary arteries?" [38] Trained interviewers conducted annual telephone follow-up of any significant health events since the last JHS contact, including diagnostic tests, hospitalizations, or death. Information on cohort hospitalizations and deaths was transmitted to the medical record abstraction (MRA) unit who reviewed death certificates and hospital records to identify CVD events. Review of completed questionnaires by physicians and medical examiners/ coroners and of death certificates and hospital records were used to obtain information of hospitalized and fatal coronary heart disease (CHD) deaths [45].

\section{Potential confounders}

Potential confounders were selected based on existing research $[6,7,10]$. We used three analysis models, that built upon one another, to analyze variables that affected the relationship with CHD. In the first model, we examined associations between intake of any combination of HFCS sweetened soda and fruit drinks and CHD events (fatal and non-fatal) with the following potential confounders: age, sex, history of smoking (yes/no), and time-varying/ time-updated covariates including BMI (continuous variable), hsCRP-a sensitive quantification of $\mathrm{c}$ reactive protein (CRP), an acute-phase protein released into the blood by the liver during inflammation (continuous variables), hypertension status (yes/no) defined as taking antihypertensive medication, or systolic BP > 140-159 or diastolic BP >90-99 $\mathrm{mmHg}$; type 2 diabetes, defined as no-diabetes (FPG $<100 \mathrm{mg} / \mathrm{dL}$ [5.6 $\mathrm{mmol} / \mathrm{l}]$ ); pre-diabetes (FPG $\geq 100 \mathrm{mg} / \mathrm{dl}$ and $\leq 125 \mathrm{mg} /$ dl $[6.9 \mathrm{mmol} / \mathrm{l}])$ ); and diabetes (FPG $\geq 126 \mathrm{mg} / \mathrm{dL}$ or use of diabetes medication (self-reported) within 2 weeks prior to the clinic visit). We also adjusted for daily total energy intake in kilocalories (a continuous variable) and physical activity level, a categorical variable, defined as physical activity levels associated with ideal, intermediate and poor health, as defined by the American Heart Association. Total energy intake was carried forward from 
exam one to exams two and three, as dietary data were collected only once, during enrollment (exam 1). Physical activity level for exam 2 was carried forward from exam 1, as it was collected only during exams 1 and 3 . For missing data (consistent with prior research) [6], the last value was carried forward for BMI, and smoking.

In the second model, we replaced diabetes status with glycated hemoglobin (HbA1c), a time-varying covariate that is a measure of diabetes control, defined as normal $(\mathrm{HbA} 1 \mathrm{c}<5.6 \%[38.8 \mathrm{mmol} / \mathrm{mol}])$, at risk $=\mathrm{HbA} 1 \mathrm{c} \geq 5.6 \%$ $(38.8 \mathrm{mmol} / \mathrm{mol})$ to $<6.5 \%[47.5 \mathrm{mmol} / \mathrm{mol}]$, or out of control $(\mathrm{HbA} 1 \mathrm{c} \geq 6.5 \%[47.5 \mathrm{mmol} / \mathrm{mol}]$, as we were interested in assessing associations between high excessfree-fructose beverages and CHD, independent of glycemic load. Given our hypothesis, that fructose malabsorption is an independent risk factor associated with $\mathrm{CHD}$, we anticipated that greater intake of non-diet soda and fruit drinks would increase risk of CHD, independent of blood glucose status and fasting serum triglycerides-an end-product of fructose metabolism. We also adjusted for low density lipoprotein-cholesterol (LDL-c), fasting serum triglycerides (continuous variables), and socio-economic status (SES)-by adjusting for education (some high school; high school graduate/ or general education development (GED) diploma; attended vocational school, trade school or college).

In the third analysis model, we replaced LDL-c and fasting serum triglycerides (non-significant predictors) with the 2010 Healty Eating Index score, a measure of diet quality, that has been described in detail elsewhere $[46,47]$. We also adjusted for intake frequency of $100 \%$ orange juice and other $100 \%$ juices, as we were interested in assessing associations between intake of any combination of HFCS sweetened soda and fruit drinks (ttlEFF), independent of these other sugar containing beverages. Conversely, analyses of $100 \%$ orange and other juices were adjusted for the other beverages. For example, the orange juice analysis was adjusted for intake of other $100 \%$ juices, and any combination of HFCS sweetened soda and fruit drinks.

\section{Statistical analysis}

There were 4815 participants at baseline, after exclusion for missing dietary intake data (237) and implausible energy intake (254), defined as total daily energy intake $\leq 600$ or $\geq 4800 \mathrm{kcal}$. There were 400 exclusions due to pre-existing CHD. Health data from three exams (9/ $2000-3 / 2004 ; 10 / 2005-12 / 2008 ; 2 / 2009-1 / 2013)$ were available for analysis. For this study, there were between 3407 and 3621 participants, aged 21-93 y (mean 55 y), depending upon analysis model, with complete responses (non-missing) to the study questions of interest. A flow-chart showing exclusions is provided as Fig. 1.
We examined incident CHD over approximately 8 years of follow-up, using multivariable-adjusted Cox proportional hazards models $(1-3)$ to estimate hazard ratios (HR). Time in the study from baseline was used for the time scale. Time varying covariates were updated at each exam. Adherence to the proportional hazards assumption was tested/confirmed using Schoenfeld and scaled Schoenfeld residuals for the models as a whole and individually, including plots for each predictor $(P \geq 0.05)$. Person-time was calculated from baseline (2005-2008) until exam 3 (2009-2013), death, loss to follow-up, or incident CHD. Stata version 13.1 was used for the analysis and a two-tailed $p$-value $<0.05$, with $95 \%$ confidence intervals that did not include 1 , was considered statistically significant.

\section{Results}

\section{Participant baseline characteristics}

At baseline, 34\% of particpants were male, $66.7 \%$ attended college ( $\geq$ some college/ college graduate), and $65.4 \%$ had above average incomes ( $\geq$ upper-middle/ affluent). Average daily energy intake was $2066 \mathrm{kcal}$ (standard deviation (SD) \pm 883$)$ and mean body mass index (BMI) was $31.8 \pm$ 7.2. More than half (54.4\%) had LDL-c concentration < $130 \mathrm{mg} / \mathrm{dL}$ (desirable target), $85.2 \%$ had fasting triglycerides within the normal range $(<150 \mathrm{mg} / \mathrm{dL})$, and $98.2 \%$ had hsCRP (inflammation biomarker) within the normal/ desirable range $(<3 \mathrm{mg} / \mathrm{L})$. Nearly a fifth $(18.5 \%)$ had hypertension and/or were taking hypertension medication; 16.7\% had diabetes; $49.7 \%$ had hbA1c above normal; and 46.7\% had an AHA physical activity level associated with sedentarism/poor health. (Table 1).

The proportion of participants who consumed orange juice (31.1\%) and non-diet soda (35.6\%) $\geq$ once per day was comparable, relative to fruit drinks (24.9\%), other $100 \%$ juices (16.9\%), and diet soda (10\%). Sixty percent of participants consumed any combination of HFCS sweetened soda and fruit drinks $\geq$ once/day. Nearly $40 \%$ consumed these beverages $>$ once/day (Table 1). The proportions of participants with $\mathrm{BMI} \geq 30 \mathrm{~kg} / \mathrm{m}$ (51-54\%), history of smoking (25-31\%), hypertension (17-21\%), or elevated LDL-c $(\geq 130 \mathrm{mg} / \mathrm{dL}$ ) were comparable across all sugar containing beverages. Participants with income below the poverty line were slightly more likely to consume HFCS sweetened soft drinks and fruit drinks than orange juice. Higher consumption of all sugar containing beverages was associated with higher total energy intake. Frequent consumers $(\geq 3 / d)$ of any combination of HFCS sweetened soft drinks and fruit drinks had the lowest (poorest) mean 2010 Healthy Eating Index score (56) (Table 2).

\section{Relationship with CHD}

There were 62 new CHD cases (after 400 exclusions due to pre-existing $\mathrm{CHD}$ ). There were no $\mathrm{CHD}$ associations 


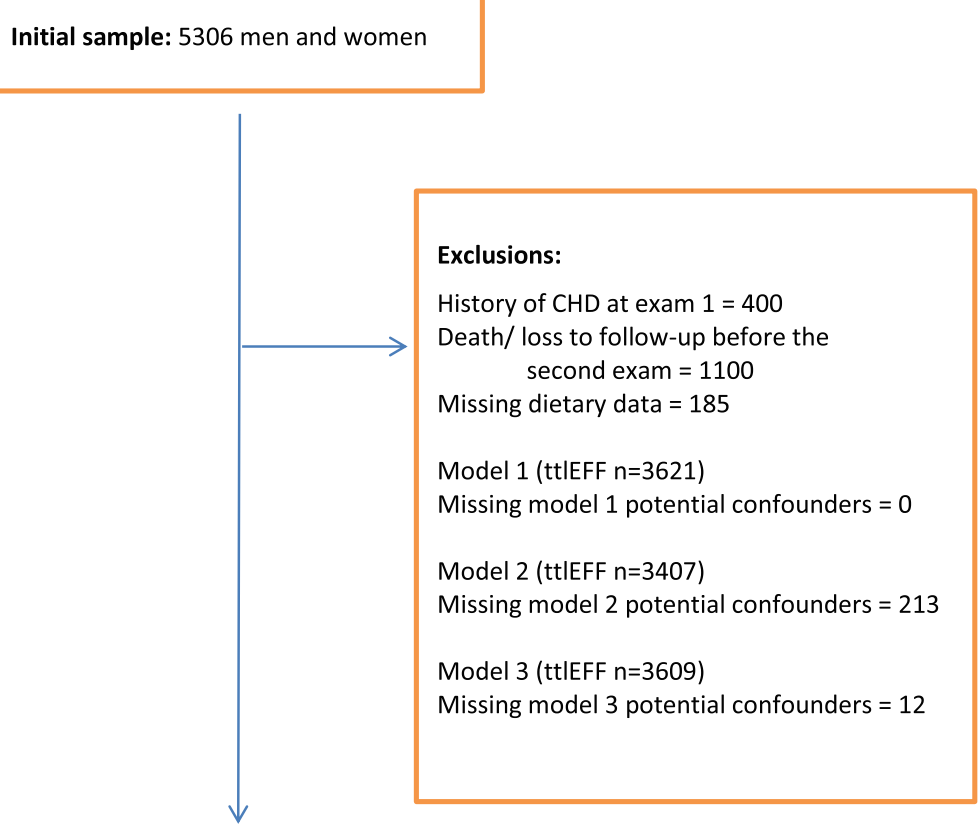

Sample for CHD analysis:

Model 1 - 3621

Model 2-3407

Model 3 - 3609

Fig. 1 Flow Chart showing Exclusions and Sample Sizes

with intake of $100 \%$ orange juice, other $100 \%$ juices (Table 3), or diet soda (data not shown). Consumers of HFCS sweetened soda 5-6 times/wk. had a 103\% higher risk of CHD, relative to seldom/ never consumers, independent of smoking history, age, sex, BMI, total energy intake, inflammation (hsCRP concentration), blood pressure, physical activity level, and type 2 diabetes (HR 2.03, 95\% CI 1.02-4.08, $p=0.045$ ) (model 1 analysis, $n=$ 3621). The association remained significant (HR 2.12, 95\% CI 1.01-4.48, $p=0.048$ ) after further adjustment for LDL-c, fasting serum triglycerides, education level, and substitution of type 2 diabetes status with blood glucose concentration-HbA1C (model 2, $n=3407$ ). The HR increased nominally, after replacement of non-significant predictors (LDL-c, fasting serum triglycerides, and SES) with 2010 Healthy Eating Index score, and intake of $100 \%$ orange and other $100 \%$ juices (HR 2.08, 95\% CI 1.03-4.20, $p=0.041$ ) (model 3, $n=3609$ ) (Table 3).

Individual analyses with fruit drinks were not significant, however $P$ for trend (Model 3) was significant. Consumption of any combination of HFCS sweetened soda and/or fruit drinks $\geq 3$ times/day was significantly associated with greater than 2.5 times higher CHD risk in all three analysis models, relative to seldom/never consumers (Model 1: HR 2.60, 95\% CI 1.07-6.31; $P=$ 0.035) (Model 2: HR 2.83, 95\% CI 1.04-7.71; $P=0.042$ ) (Model 3: HR 2.98, 95\% CI 1.15-7.76; $P=0.025$ ). The association with CHD was independent of age, sex, smoking history, BMI, total energy intake, inflammation (hsCRP concentration), hypertension, physical activity level, type 2 diabetes status (model 1 analyses). It was independent of LDL-c, fasting serum triglyceride, HbA1c, and education level (model 2 analyses); and it was independent of diet quality, as measured by the 2010 Healthy Eating Index score (model 3 analyses). $P$ for trend was also significant (Model 3).

Hypertension and inflammation were associated with CHD across all analysis models (1-3). Type 2 diabetes approached significance. The American Heart Association's (AHA) physical activity level associated with ideal health was protective $(\geq 150 \mathrm{~min} / \mathrm{wk}$. moderate; or $\geq 75$ $\mathrm{min} / \mathrm{wk}$. vigorous, $\geq 150 \mathrm{~min} / \mathrm{wk}$. moderate, $+2 \mathrm{x}$ vigorous; $\geq 60$ of moderate or vigorous every day) across all analysis models (1-3), independent of potential confounders. 
Table 1 Baseline characteristics of adults included in this analysis, aged $\geq 21$ years, in the Jackson Heart Study ${ }^{a}$

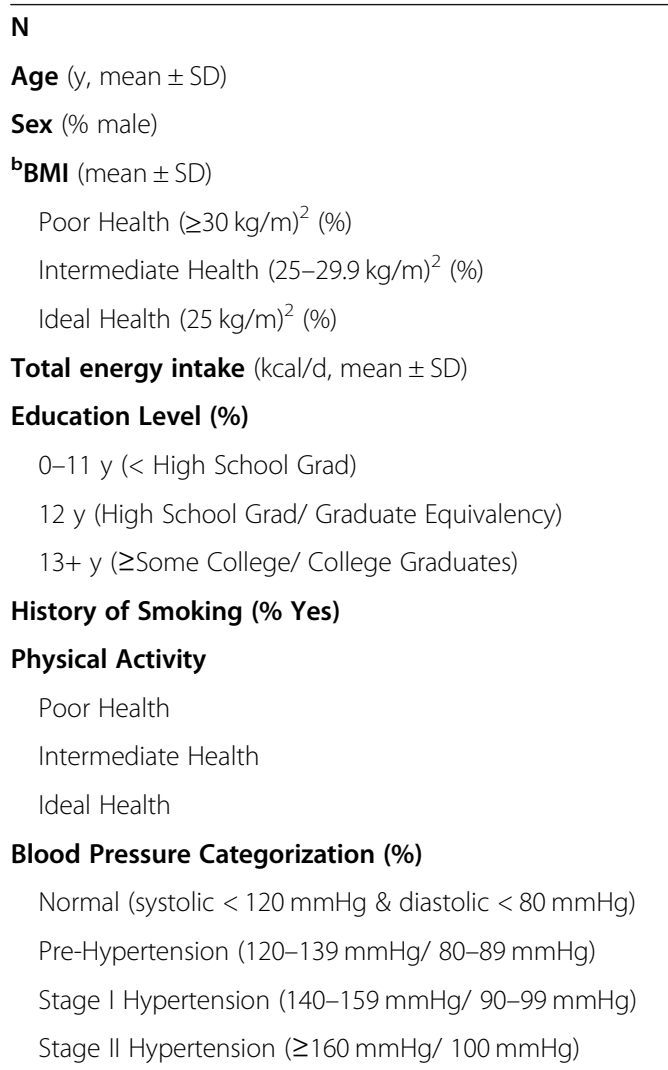

3233-3533

$55.0 \pm 12.2$

33.8

$31.8 \pm 7.2$

53.3

33.1

13.6

$2065 \pm 883$

16.2

17.1

66.7

28.9

46.7

33.4

19.9

32.5

49.0

14.9

3.6

Fasting Low Density Lipoprotein (LDL) (\%)

Optimal $(<100 \mathrm{mg} / \mathrm{dL})$

Near/ Above Optimal (100-129 mg/dL)

Borderline High (130-159 mg/dL)

High (160-189 mg/dL)

Very High $(190 \mathrm{mg} / \mathrm{dL})$

Fasting High Density Lipoprotein (HDL) (\%)

Low (< $40 \mathrm{mg} / \mathrm{dL}$ high risk)

Normal (40-59 mg/dL)

High ( $\geq 60 \mathrm{mg} / \mathrm{dL}$ )

\section{Fasting Triglyceride Categorization (\%)}

Normal (normal $(<150 \mathrm{mg} / \mathrm{dL}$ )

Borderline High (150-199 mg/dL)

High ( $\geq 200 \mathrm{mg} / \mathrm{dL}$ )

\section{hsCRP (\%)}

Low risk $<1 \mathrm{mg} / \mathrm{L}$

Average risk $1-3 \mathrm{mg} / \mathrm{L}$

High risk $>3 \mathrm{mg} / \mathrm{L}$

\section{Diabetes Classification (\%)}

No-Diabetes

Pre-Diabetes

Diabetes
Table 1 Baseline characteristics of adults included in this analysis, aged $\geq 21$ years, in the Jackson Heart Study ${ }^{a}$ (Continued) Hemoglobin A1c (\%)

$\begin{array}{ll}\text { Normal }(<5.6 \%(38.8 \mathrm{mmol} / \mathrm{mol}) & 50.3 \\ \text { At Risk }(\geq 5.6 \%(38.8 \mathrm{mmol} / \mathrm{mol}) & 36.0\end{array}$

Intake frequency cany combination non-diet soda and fruit drinks (ttIEFF) (\%)

$\begin{array}{ll}<2 \text { times } \mathrm{p} / \mathrm{wk} & 19.2 \\ 2-5 \text { times } \mathrm{p} / \mathrm{wk} & 20.3 \\ \text { once } \mathrm{p} / \text { day } & 21.1 \\ >1 \text { to }<3 \text { times } \mathrm{p} / \text { day } & 20.9 \\ \geq 3 \text { times } \mathrm{p} / \text { day } & 18.5\end{array}$

Intake frequency non-diet soda (\%)

$$
\text { sonce /wk }
$$

2-4 times /wk

5-7 times /wk

$>$ once / day

Intake frequency fruit drinks (\%)

sonce /wk

2-4 times /wk

5-7 times /wk

$>$ once /day

Intake frequency orange juice (\%)

$$
\begin{array}{ll}
\text { s once /wk } & 25.4 \\
2-4 \text { times /wk } & 26.9 \\
\geq 5 \text { times /wk. } & 16.6 \\
>\text { once /day } & 31.1
\end{array}
$$

Intake frequency other $100 \%$ juices $^{d}(\%)(n=3425)$

$$
\begin{array}{ll}
\text { S once /wk } & 49.4 \\
\text { 2-4 times /wk } & 19.2 \\
\geq 5 \text { times /wk } & 14.5 \\
\text { > once /day } & 16.9
\end{array}
$$

Intake frequency diet soda (\%)

$$
\text { sonce /wk } \quad 81.6
$$$$
2-7 \text { times /wk } 8.1
$$$$
\text { > once /day } 10.3
$$

2010 Healthy Eating Index, mean score 64.3

\pm SD

11.1

${ }^{a}$ Characteristics are based upon self-reported responses to the medical and food frequency questionnaires administered at baseline (10/2005-12/2008). ${ }^{b}$ American Heart Association classifications for body mass index (BMI). ${ }^{c} \mathrm{ttlEFF}$ refers to any combination of high fructose corn syrup sweetened soft drinks, and fruit drinks. ${ }^{d}$ Other juices excluded citrus juice (100\% orange juice) and included apple (a high fructose juice), grape, pineapple, and other $100 \%$ juices

Serum LDL-c concentration was not associated with CHD in any of the analysis models, which is consistent with existing JHS research [48]; nor were BMI, total energy intake, SES, or fasting serum triglyceride concentration-a 
Table 2 Baseline Characteristics of Participants by Beverage Intake Frequency in the Jackson Heart Study ${ }^{a}$

\begin{tabular}{|c|c|c|c|c|c|c|c|c|c|c|c|c|c|}
\hline \multicolumn{6}{|c|}{ ttIEFF - Any Combo of HFCS sweetened Soft Drinks, and Fruit Drinks } & \multicolumn{4}{|c|}{ Orange Juice } & \multicolumn{4}{|c|}{ Other $100 \%$ Juices } \\
\hline \multicolumn{14}{|c|}{ Intake Frequencies } \\
\hline & $<2 /$ wk & $2-5 / w k$ & $1 / d$ & $>1$ to $<3 /$ d & $\geq 3 / d$ & $\leq 1 /$ wk & $2-4 / w k$ & $5-7 / w k$ & $>1 / d$ & $\leq 1 /$ wk & $2-4 / w k$ & $5-7 / w k$ & $>1 / c$ \\
\hline $\mathbf{N}$ & 699 & 728 & 738 & 727 & 641 & 897 & 951 & 588 & 1097 & 1760 & 671 & 517 & 585 \\
\hline Mean Age (y) & 58 & 56 & 54 & 54 & 51 & 55 & 53 & 55 & 56 & 55 & 52 & 57 & 55 \\
\hline Gender (male \%) & 25 & 33 & 37 & 35 & 39 & 24 & 40 & 34 & 36 & 32 & 43 & 24 & 38 \\
\hline \multicolumn{14}{|l|}{ Smoking History } \\
\hline Yes (\%) & 30 & 28 & 25 & 30 & 31 & 31 & 30 & 25 & 27 & 31 & 26 & 27 & 28 \\
\hline \multicolumn{14}{|l|}{ bBMI Health(\%) } \\
\hline Poor ( $\geq 30 \mathrm{~kg} / \mathrm{m})$ & 55 & 53 & 53 & 53 & 53 & 55 & 51 & 53 & 54 & 54 & 51 & 52 & 54 \\
\hline Intermediate $(25-29.9 \mathrm{~kg} / \mathrm{m})$ & 35 & 34 & 33 & 33 & 32 & 32 & 33 & 35 & 33 & 32 & 35 & 33 & 34 \\
\hline Ideal (18.5-25 kg/m) & 10 & 13 & 14 & 15 & 15 & 12 & 16 & 12 & 14 & 13 & 13 & 15 & 13 \\
\hline \multicolumn{14}{|l|}{ Mean Total Energy Intake } \\
\hline daily kilocal & 1591 & 1814 & 1976 & 2256 & 2716 & 1867 & 1991 & 2070 & 2288 & 1907 & 2146 & 2063 & 2445 \\
\hline \multicolumn{14}{|l|}{2010 Healthy Eating Index } \\
\hline Mean score & 70 & 68 & 65 & 63 & 56 & 62 & 62 & 66 & 67 & 63 & 64 & 67 & 67 \\
\hline \multicolumn{14}{|l|}{ Education Level (\%) } \\
\hline $0-11$ y & 19 & 16 & 12 & 16 & 18 & 16 & 17 & 15 & 17 & 18 & 16 & 15 & 13 \\
\hline $12 y$ & 16 & 15 & 18 & 18 & 19 & 20 & 16 & 14 & 17 & 18 & 15 & 17 & 18 \\
\hline $13+y$ & 65 & 69 & 70 & 66 & 63 & 64 & 67 & 71 & 66 & 64 & 70 & 68 & 69 \\
\hline \multicolumn{14}{|l|}{ 'Income (\%) } \\
\hline Low & 12 & 11 & 10 & 12 & 14 & 14 & 13 & 09 & 11 & 12 & 10 & 14 & 10 \\
\hline Middle & 51 & 51 & 51 & 53 & 56 & 51 & 54 & 53 & 53 & 54 & 53 & 56 & 52 \\
\hline Affluent & 36 & 37 & 38 & 34 & 27 & 30 & 36 & 37 & 36 & 34 & 37 & 30 & 38 \\
\hline \multicolumn{14}{|l|}{ Physical Activity Health (\%) } \\
\hline Poor & 42 & 45 & 44 & 50 & 51 & 51 & 47 & 41 & 46 & 48 & 45 & 45 & 44 \\
\hline Intermediate & 37 & 32 & 34 & 30 & 34 & 34 & 33 & 36 & 32 & 33 & 34 & 33 & 33 \\
\hline |deal & 21 & 23 & 21 & 19 & 15 & 16 & 20 & 23 & 22 & 18 & 21 & 22 & 22 \\
\hline
\end{tabular}

hsCRP (\%)

\begin{tabular}{|c|c|c|c|c|c|c|c|c|c|c|c|c|}
\hline Low risk< $1 \mathrm{mg} / \mathrm{L}$ & 89 & 89 & 89 & 90 & 88 & 89 & 84 & 90 & 90 & 89 & 90 & 90 \\
\hline Avg risk $1-3 \mathrm{mg} / \mathrm{L}$ & 9 & 9 & 10 & 9 & 9 & 9 & 9 & 9 & 8 & 9 & 8 & 9 \\
\hline High risk $>3 \mathrm{mg} / \mathrm{L}$ & 2 & 2 & 1 & 1 & 3 & 2 & 2 & 1 & 2 & 2 & 2 & 1 \\
\hline
\end{tabular}

\section{Hypertension}

$$
\text { (Yes\%) }
$$

Fasting LDL (\%)

\begin{tabular}{|c|c|c|c|c|c|c|c|c|c|c|c|c|}
\hline Borderline High & 25 & 29 & 30 & 28 & 25 & 26 & 29 & 25 & 28 & 29 & 26 & 26 \\
\hline High & 10 & 13 & 13 & 16 & 12 & 12 & 13 & 11 & 15 & 12 & 14 & 13 \\
\hline Very High & 5 & 5 & 5 & 6 & 4 & 6 & 5 & 4 & 4 & 5 & 5 & 5 \\
\hline
\end{tabular}

Fasting HDL (\%)

$$
\text { Low }
$$$$
\begin{array}{llll}
34 & 34 & 34 & 40
\end{array}
$$$$
\begin{array}{lll}
37 & 38 \quad 37
\end{array}
$$$$
35
$$$$
34 \quad 36
$$$$
\begin{array}{lll}
37 & 36 \quad 35
\end{array}
$$

Fasting Triglyceride (\%)

$$
\begin{array}{llllllll}
\text { Borderline High } & 8 & 10 & 8 & 12 & 10 & 9 \\
\text { High } & 5 & 6 & 5 & 4 & 5 & 5 & 5 \\
\text { Diabetes (Yes \%) } & 32 & 17 & 14 & 11 & 10 & 18
\end{array}
$$

$\begin{array}{llll}8 & 10 & 8 & 12 \\ 5 & 6 & 5 & 4\end{array}$

$\begin{array}{llll}32 & 17 & 14 & 11\end{array}$

${ }^{\mathrm{a}}$ Characteristics by beverage intake frequency are based upon self-reported responses to the medical and food frequency questionnaires administered at baseline (10/2005-12/2008). ${ }^{b}$ American Heart Association classifications for body mass index (BMI). ' Income is classified as (low income (<poverty level); lower-middle (1-1.6 times the poverty level); upper-middle (1.6-3.5 times the poverty level); affluent (at least 3.5 times the poverty level) 
Table 3 Coronary Heart Disease ${ }^{a}$ Risk according to Beverage Consumption in Adults, The Jackson Heart Study

\begin{tabular}{|c|c|c|c|c|c|c|c|c|c|c|c|c|c|}
\hline & \multicolumn{3}{|c|}{$\begin{array}{l}\text { Cox Proportional Hazards } \\
\text { Model } 1, n=3621\end{array}$} & \multicolumn{3}{|c|}{$\begin{array}{l}\text { Cox Proportional Hazards } \\
\text { Model } 2, n=3407\end{array}$} & \multicolumn{3}{|c|}{$\begin{array}{l}\text { Cox Proportional Hazards } \\
\text { Model } 3, n=3609\end{array}$} & \multirow{2}{*}{$\begin{array}{l}\text { P } \\
\text { for } \\
\text { Trend }\end{array}$} & \multirow{2}{*}{$\begin{array}{l}\text { IR } \\
\text { per } \\
(1000)\end{array}$} & \multirow{2}{*}{$\begin{array}{l}\text { Person } \\
\text { Time } \\
\text { Years }\end{array}$} & \multirow{2}{*}{$\begin{array}{l}\text { Cases } \\
\mathrm{p} / \\
1000 \\
\mathrm{p} / \\
\text { year }\end{array}$} \\
\hline & $\begin{array}{l}\text { Hazard } \\
\text { Ratio }\end{array}$ & $\begin{array}{l}95 \% \\
\mathrm{Cl}\end{array}$ & $\begin{array}{l}\mathrm{p}- \\
\text { value }\end{array}$ & $\begin{array}{l}\text { Hazard } \\
\text { Ratio }\end{array}$ & $\begin{array}{l}95 \% \\
\mathrm{Cl}\end{array}$ & $\begin{array}{l}\mathrm{p}- \\
\text { value }\end{array}$ & $\begin{array}{l}\text { Hazard } \\
\text { Ratio }\end{array}$ & $\begin{array}{l}95 \% \\
\mathrm{Cl}\end{array}$ & $\begin{array}{l}\mathrm{p}- \\
\text { value }\end{array}$ & & & & \\
\hline & \multicolumn{3}{|c|}{$\begin{array}{l}\text { HR-adjusted for ever } \\
\text { smoker, age, sex, and time } \\
\text { varying covariates -BMI, } \\
\text { total energy intake, hsCRPd, } \\
\text { hypertension, physical } \\
\text { activity type } 2 \text { diabetes }\end{array}$} & \multicolumn{3}{|c|}{$\begin{array}{l}\text { HR- adjusted for ever } \\
\text { smoker, age, sex, SES, \& } \\
\text { time varying covariates-BMl, } \\
\text { total energy intake, hsCRPd, } \\
\text { hypertension, physical activ- } \\
\text { ity, hbA1ce, fasting serum } \\
\text { triglycerides, LDL-c }\end{array}$} & \multicolumn{3}{|c|}{$\begin{array}{l}\text { HR- adjusted for ever } \\
\text { smoker, age, sex, orange } \\
\text { juice, and other 100\% juice } \\
\text { intake, HEI2010, SES, \& time } \\
\text { varying covariates-BMl, total } \\
\text { energy intake, hsCRPd, } \\
\text { hypertension, physical activ- } \\
\text { ity, hbA1c }\end{array}$} & & & & \\
\hline \multicolumn{14}{|c|}{${ }^{\mathrm{b}}$ Any combination of ndsoda/ FD (ttIEFF) } \\
\hline$<2$ times $\mathrm{p} / \mathrm{wk}$ & Reference & & & Reference & & & Reference & & & & 10 & 4097 & 2.4 \\
\hline $2-5$ times $p / w k$ & 0.95 & $\begin{array}{l}0.38- \\
2.36\end{array}$ & 0.910 & 1.35 & $\begin{array}{l}0.49- \\
3.67\end{array}$ & 0.560 & 1.08 & $\begin{array}{l}0.43- \\
2.73\end{array}$ & 0.871 & & 9 & 4338 & 2.1 \\
\hline once p/day & 1.79 & $\begin{array}{l}0.78- \\
4.09\end{array}$ & 0.168 & 2.16 & $\begin{array}{l}0.85- \\
5.51\end{array}$ & 0.108 & 2.00 & $\begin{array}{l}0.86- \\
4.91\end{array}$ & 0.129 & & 15 & 4493 & 3.3 \\
\hline$>1$ to $<3 \mathrm{X}$ p/day & 1.77 & $\begin{array}{l}0.76- \\
4.16\end{array}$ & 0.187 & 1.89 & $\begin{array}{l}0.70- \\
5.06\end{array}$ & 0.207 & 2.03 & $\begin{array}{l}0.84- \\
5.19\end{array}$ & 0.117 & & 15 & 4865 & 3.1 \\
\hline$>=3$ times $p /$ day & 2.60 & $\begin{array}{l}1.07- \\
6.31\end{array}$ & $0.035^{*}$ & 2.83 & $\begin{array}{l}1.04- \\
7.71\end{array}$ & $0.042^{*}$ & 2.98 & $\begin{array}{l}1.15- \\
7.76\end{array}$ & $0.025^{*}$ & $0.013^{*}$ & 17 & 4891 & 3.5 \\
\hline
\end{tabular}

\section{Non-diet soda}

\pm beverage adjustment $100 \%$ orange juice, and other $100 \%$ juices

\begin{tabular}{|c|c|c|c|c|}
\hline$\leq$ once /wk & Reference & & & Reference \\
\hline 2-4 times /wk & 1.78 & $\begin{array}{l}0.77- \\
4.08\end{array}$ & 0.174 & 1.63 \\
\hline 5-6 times /wk & 2.03 & $\begin{array}{l}1.02- \\
4.08\end{array}$ & $0.045^{*}$ & 2.12 \\
\hline$\geq$ once /day & 1.34 & $\begin{array}{l}0.70- \\
2.55\end{array}$ & 0.379 & 1.20 \\
\hline
\end{tabular}

\section{Fruit Drinks}

\begin{tabular}{|c|c|c|c|c|}
\hline$\leq$ once /wk & Reference & & & Reference \\
\hline 2-4 times /wk & 0.44 & $\begin{array}{l}0.19- \\
1.02\end{array}$ & 0.056 & 0.54 \\
\hline 5-6 times $p / w k$ & 1.60 & $\begin{array}{l}0.81- \\
3.17\end{array}$ & 0.176 & 1.58 \\
\hline$\geq$ once /day & 1.61 & $0.81-$ & 0.177 & 1.50 \\
\hline
\end{tabular}

\section{Orange Juice}

\begin{tabular}{|c|c|c|c|c|}
\hline$\leq$ once /wk & Reference & & & Reference \\
\hline 2-4 times /wk & 1.20 & $\begin{array}{l}0.60- \\
2.40\end{array}$ & 0.602 & 1.22 \\
\hline 5-6 times /wk & 0.76 & $\begin{array}{l}0.33- \\
1.78\end{array}$ & 0.529 & 0.92 \\
\hline$\geq$ once /day & 1.28 & $\begin{array}{l}0.66- \\
2.50\end{array}$ & 0.468 & 1.27 \\
\hline
\end{tabular}

COther $100 \%$ (noncitrus) Juices

Reference

\begin{tabular}{|c|c|c|c|c|c|c|c|}
\hline & & Reference & & & & 21 & 8542 \\
\hline $\begin{array}{l}0.66- \\
4.00\end{array}$ & 0.289 & 1.70 & $\begin{array}{l}0.73- \\
3.96\end{array}$ & 0.215 & & 8 & 2294 \\
\hline $\begin{array}{l}1.01- \\
4.48\end{array}$ & $0.048^{*}$ & 2.08 & $\begin{array}{l}1.03- \\
4.20\end{array}$ & $0.041^{*}$ & & 14 & 3347 \\
\hline $\begin{array}{l}0.60- \\
2.43\end{array}$ & 0.604 & 1.21 & $\begin{array}{l}0.61- \\
2.38\end{array}$ & 0.585 & 0.498 & 23 & 8501 \\
\hline
\end{tabular}

土beverage adjustment 100\% orange juice, and other 100\% juices

\begin{tabular}{|c|c|c|c|c|c|c|}
\hline & Reference & & & & 18 & 5902 \\
\hline $\begin{array}{l}0.22- \\
1.28\end{array}$ & 0.46 & $\begin{array}{l}0.18- \\
1.14\end{array}$ & 0.094 & & 8 & 6425 \\
\hline $\begin{array}{l}0.75- \\
3.33\end{array}$ & 1.72 & $\begin{array}{l}0.84- \\
3.54\end{array}$ & 0.140 & & 18 & 3917 \\
\hline 0.292 & 1.76 & $\begin{array}{l}0.85- \\
3.65\end{array}$ & 0.129 & $0.012^{*}$ & 22 & 6440 \\
\hline
\end{tabular}

土beverage adjustment/ ttIEFF/ 100\% non-citrus juices

$\begin{array}{llll}\text { Reference } & 15 & 5697 & 2.6\end{array}$

\begin{tabular}{|c|c|c|c|c|c|c|}
\hline 0.612 & 1.21 & $\begin{array}{l}0.60- \\
2.45\end{array}$ & 0.588 & & 18 & 6222 \\
\hline 0.850 & 0.78 & $\begin{array}{l}0.32- \\
1.88\end{array}$ & 0.578 & & 9 & 3572 \\
\hline 0.526 & 1.42 & $\begin{array}{l}0.71- \\
2.88\end{array}$ & 0.324 & 0.602 & 24 & 7194 \\
\hline
\end{tabular}

土beverage adjustment/ ttIEFF/ 100\% orange juice

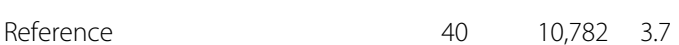


Table 3 Coronary Heart Disease ${ }^{a}$ Risk according to Beverage Consumption in Adults, The Jackson Heart Study (Continued)

\begin{tabular}{|c|c|c|c|c|c|c|c|c|c|c|c|c|c|}
\hline & \multicolumn{3}{|c|}{$\begin{array}{l}\text { Cox Proportional Hazards } \\
\text { Model } 1, n=3621\end{array}$} & \multicolumn{3}{|c|}{$\begin{array}{l}\text { Cox Proportional Hazards } \\
\text { Model } 2, n=3407\end{array}$} & \multicolumn{3}{|c|}{$\begin{array}{l}\text { Cox Proportional Hazards } \\
\text { Model } 3, n=3609\end{array}$} & \multirow{2}{*}{$\begin{array}{l}P \\
\text { for } \\
\text { Trend }\end{array}$} & \multirow{2}{*}{$\begin{array}{l}\text { IR } \\
\text { per } \\
(1000)\end{array}$} & \multirow{2}{*}{$\begin{array}{l}\text { Person } \\
\text { Time } \\
\text { Years }\end{array}$} & \multirow{2}{*}{$\begin{array}{l}\text { Case } \\
\mathrm{p} / \\
1000 \\
\mathrm{p} / \\
\text { year }\end{array}$} \\
\hline & $\begin{array}{l}\text { Hazard } \\
\text { Ratio }\end{array}$ & $\begin{array}{l}95 \% \\
\mathrm{Cl}\end{array}$ & $\begin{array}{l}\mathrm{p}- \\
\text { value }\end{array}$ & $\begin{array}{l}\text { Hazard } \\
\text { Ratio }\end{array}$ & $\begin{array}{l}95 \% \\
\mathrm{Cl}\end{array}$ & $\begin{array}{l}\mathrm{p}- \\
\text { value }\end{array}$ & $\begin{array}{l}\text { Hazard } \\
\text { Ratio }\end{array}$ & $\begin{array}{l}95 \% \\
\mathrm{Cl}\end{array}$ & $\begin{array}{l}\mathrm{p}- \\
\text { value }\end{array}$ & & & & \\
\hline & ------ & & & ------ & & & ------ & & & & & & \\
\hline 2-4 times /wk & 0.43 & $\begin{array}{l}0.18- \\
1.02\end{array}$ & 0.056 & 0.48 & $\begin{array}{l}0.20- \\
1.15\end{array}$ & 0.098 & 0.42 & $\begin{array}{l}0.18- \\
1.02\end{array}$ & 0.054 & & 6 & 4993 & 1.2 \\
\hline 5-6 times /wk & 0.89 & $\begin{array}{l}0.45- \\
1.74\end{array}$ & 0.732 & 0.60 & $\begin{array}{l}0.27- \\
1.34\end{array}$ & 0.213 & 0.96 & $\begin{array}{l}0.48- \\
1.94\end{array}$ & 0.910 & & 12 & 2960 & 4.0 \\
\hline$\geq$ once /day & 0.55 & $\begin{array}{l}0.25- \\
1.21\end{array}$ & 0.136 & 0.47 & $\begin{array}{l}0.20- \\
1.10\end{array}$ & 0.080 & 0.45 & $\begin{array}{l}0.20- \\
1.02\end{array}$ & 0.055 & 0.247 & 8 & 3949 & 2.0 \\
\hline
\end{tabular}

HR hazard ratios (relative risks), their $95 \%$ confidence intervals and $p$ values are shown. ${ }^{a}$ Coronary Heart Disease (CHD) refers to the first incidence of CHD as selfreported during exams 2-3. ${ }^{b} \mathrm{HR}$ includes adjustment for any combination of HFCS sweetened soft drinks (ndSoda), and fruit drinks (FD)-beverages with fructoseto-glucose ratios that exceed 1:1. During the Jackson Heart Study -HFCS was the main sweetener in US soft drinks [41]. In HFCS sweetened soft drinks, independent labs have found that the fructose-to-glucose ratio (1.9:1 [35] and 1.5:1 [36]) exceeds the 1.2:1 ratio that is generally recognized as safe [37]. The fructose-to-glucose ratio in fruit drinks varies and likely lies between 1.5:1 and 2:1, as many varieties are sweetened with apple juice-a 100\% juice with a $22: 1$ fructose-to-glucose ratio [11], and HFCS. ${ }^{C}$ Other $100 \%$ Juices includes $100 \%$ juices other than orange juice (a 100\% juice with a 1:1 fructose-to-glucose ratio [11]), for example pineapple and grape juices (100\% juices with a 1:1 fructose-to-glucose ratio [11]), and apple juice (a 100\% juice with a $\geq 2: 1$ fructose-to-glucose ratio [11]). ${ }^{d}$ hsCRP is a biomarker of inflammation. ${ }^{e}$ hbA1C is a measure of glycated hemoglobin, an indicator of blood glucose concentration over the prior 3 months. ${ }^{*}$ Asterisks indicate statistical significance. ${ }^{ \pm}$specifies which other beverages were included in model 3 analyses

metabolic end-product of fructose metabolism (data not shown).

There was an increasing pattern of CHD cases, per 1000 persons, per year, with increasing consumption of HFCS sweetened soda and fruit drinks. The pattern was flat across the spectrum of orange juice intake. There was no consistent pattern with intake of other $100 \%$ non-citrus juices (Table 3).

\section{Discussion}

African Americans who regularly consumed HFCS sweetened soda and/or fruit drinks had significantly higher CHD risk, independent of CHD risk factors, including age, sex, socio-economic status, history of smoking, elevated serum LDL cholesterol, inflammation, hypertriglyceridemia, hypertension, sedentary lifestyles, unhealthy BMI, type 2 diabetes, and elevated blood glucose, than seldom/never consumers. Participants who consumed HFCS sweetened soda almost every day (5-6 times $\mathrm{p} / \mathrm{wk}$ ) had two times the CHD risk, and participants who consumed any combination of HFCS sweetened soda and fruit drinks $\geq 3$ times/day had more than 2.5-3 times the CHD risk, of seldom/ never consumers. There were no associations with intake of diet soda, $100 \%$ orange juice, or other $100 \%$ juices. The significantly higher CHD risk borne by African Americans, who regularly consumed any combination of HFCS sweetened soda and fruit drinks (high excess-freefructose beverages) and the negative findings with orange juice, other $100 \%$ juices, and diet soda, support our hypothesis. The CHD association appears to be with the high fructose-to-glucose ratios - not with all forms of fructose. Glycemic load does not explain the CHD associations, as orange juice has a glycemic load (15) that is marginally lower than non-diet cola (16), per $250 \mathrm{ml}$ serving [12]. It is also noteworthy that the difference in total fructose, between orange juice (11 g per $8 \mathrm{oz}$. cup) [11] and apple juice (15.7 per 8 oz. cup) [11] - a $100 \%$ juice with $\mathrm{a} \geq 2: 1$ fructose-to-glucose ratio [11], previously associated with CHD [10] - is nominal.

At baseline, $85.2 \%$ of participants had normal $(<150$ $\mathrm{mg} / \mathrm{dL}$ ) fasting triglyceride concentration, despite the fact that $60.5 \%$ reported intake of any combination of HFCS sweetened soda and fruit drinks $\geq$ once per day. This is unexpected in a cohort wherein a high percentage of participants $(60.5 \%)$ reported frequent ( $\geq$ once per day) intake of HFCS sweetened soda and fruit drinks, as there is consistent evidence that increased fructose intake increases fasting/postprandial triglycerides, and LDL-C (dyslipidemia) [49, 50]. Fructose does not appear to be following the normal pathway of metabolism. These results are consistent with research which found that African Americans have higher fructose malabsorption prevalence than Hispanics, despite both groups having high dietary fructose/SSB intake profiles [31]. Higher fructose malabsorption prevalence may contribute to the different atherogenic profile (lower visceral adipose tissue/serum free fatty acid concentrations) and different diabetogenic profile (impaired insulin secretion) [51] seen in non-Hispanic blacks relative to non-Hispanic whites. The high percentage of participants with normal fasting triglycerides is consistent with the possibility that the CHD association is, in part, with gut-heart mechanisms that stem from unabsorbed fructose and less so with fructose metabolism by the liver [13, 28-30].

The high magnitude of association between regular intake of high excess-free-fructose beverages and increased 
CHD risk, among African Americans in the JHS, parallels our cross sectional analysis results with nationally representative survey data (NHANES) [10]. Both results are higher than associations observed in existing large scale longitudinal studies $(n=42,883$ [6]; $n=88,250$ [7]) of soft drinks and CHD with mainly non-Hispanic white adults. Notably, in prior analyses with NHANES [10], and in the Health Professionals Follow-Up Study $(n=42$, 883) [6], associations with CHD persisted, independent of hypertension, and serum triglyceride, glucose $[6,10]$, and cholesterol concentrations [10]. Results from these studies are consistent with the possibility that the CHD association is with gut-heart mechanisms that precede fructose metabolism [13, 28-30].

Industry sponsored messaging has promoted the viewpoint that HFCS is chemically similar to, and no more problematic to health and wellness than table sugar (sucrose) [52-54]. However, these reports have overlooked the fact that the fructose used to sweeten popular US sodas has contained fructose-to-glucose ratios (1.9-to-1 [35] and 1.5-to-1 [36]) that are significantly higher than the 1.2-to-1 ratio that is GRAS [37]. In $65 \mathrm{~g}$ of HFCS (average per capita consumption below peak (80 g)) [23$25]$ that is $55 \%$ fructose, there are $6.4 \mathrm{~g}$ of excess-freefructose. When the fructose content increases to $60 \%$, the amount of excess-free-fructose doubles to $13 \mathrm{~g}$. When the fructose content increases to $65 \%$, the amount of excess-free-fructose triples to $19.4 \mathrm{~g}$. These amounts do not reflect excess-free-fructose intakes from apple juice [11], agave syrup ( $\geq 60 \%$ fructose) [55], or crystalline fructose-an increasingly popular fructose variant that is $100 \%$ fructose, or from naturally high excess-freefructose fruits, including apples, pears, watermelons and mangoes [11]. Notably, $237 \mathrm{ml}$ (one 8 oz. cup) of apple juice contributes $\sim 8 \mathrm{~g}$ of excess-free-fructose (EFF) to the daily EFF load from HFCS, whereas the EFF contribution from orange juice is low $(0.4 \mathrm{~g})$ [11]. This is problematic given that the capacity to absorb free fructose is saturable and ranges widely from $\sim 5 \mathrm{~g}$ to $>50 \mathrm{~g}$ in healthy adults [13].

The amount of free fructose in the HFCS in popular sodas was the focus of recent beverage industry sponsored research [56]. Results pointed to the presence of previously undetected maltose/small chain glucose oligomers $(5-8 \%)$. However, these findings are not relevant in the context of fructose malabsorption, as fructose malabsorption occurs after consumption of free fructose (crystalline fructose, HFCS, agave syrup, and high excess free fructose fruits) whether or not the beverage/food contains small chain glucose oligomers. In recent research, investigators assessed whether co-ingestion of glucose improved fructose absorption/fructose malabsorption symptoms after consumption of whole foods containing fructose in excess of glucose [57], as in HFCS sweetened food/beverages. Using breath hydrogen tests, they found that the addition of glucose did not improve excess-free-fructose absorption/ fructose malabsorption related symptoms [57]. This is consistent with recent research which casts doubt on GLUT2's previously reported role in glucose/fructose co-transport and coabsorption [58-61]. What appears clear is that apical SGLT1 transports glucose [58] and GLUT5 transports fructose [62]. Neither appears to be significantly involved in absorption of the other monomer [58, 62]. There is no evidence that we know of, that shows improvements in excess-free-fructose absorption with co-ingestion of maltose/short chain glucose oligomers.

Other epidemiological research is consistent with our findings. In a study of Japanese adults, soft drinks were not associated with CHD (ischemic heart disease) [63]. In Japan, HFCS output is limited by the government and accounts for less than one third of total sugar consumption [64]. In another nationally representative study (NHANES) of CVD mortality among US adults [65], there was a significant dose-dependent relationship between added sugar intake and CVD mortality across races, except among non-Hispanic blacks. The lack of association with CVD, among non-Hispanic blacks, may be due to the fact that the exposure variable, included all forms of added sugars, including non/low excess-freefructose sugars (sucrose, honey, maple syrup, i.e. sugars with a 1:1 fructose-to-glucose ratio), glucose based sugars (glucose syrup, dextrose, maltodextrin), and sugars with a high fructose-to-glucose ratio (HFCS, agave syrup, crystalline fructose) [65]. Research that disambiguates SSB by sugar type are needed.

Recent research by Hieronimus et al [66] confirmed that increases in serum triglycerides, uric acid, and apoCIII are driven mainly by the fructose fraction in sugars/unregulated metabolism of fructose. Their study led to a novel finding, wherein participants who consumed HFCS had higher increases of CHD risk factors (nonHDL-C, LDL-C, apoB) than those consuming fructose at the same level. Researchers proposed a two step hypothesis to explain their results: that co-ingestion of glucose and fructose, as in HFCS, leads to nonenzymatic glycation of LDL particles by the serum glucose component of HFCS (step 2), which interferes with the clearance of LDL particles, which are downstream products of fructose induced lipid metabolism/hydrolysis of triglyceride rich lipoproteins (step 1) [66]. Notably, fructose malabsorption status of participants was not assessed at baseline.

Existing epidmiogical studies of SSB and CHD $[6,10]$, including our findings, suggest that other mechanisms are involved, as regular consumption of HFCS sweetened beverages remained significantly associated with increased CHD risk, independent of serum glucose, triglyceride and 
LDL-C concentrations. Potential mechanisms that could explain our results include disruptions to the microbiome by unabsorbed/luminal fructose [28] that promote hypertension and atherosclerosis [29, 30], as previously described. Individuals with lower intestinal fructose absorption have higher luminal fructose concentration. Unabsorbed fructose affects bacterial load and modifies the composition of the gut microbiome [28]. Research shows that patients with small intestinal bacterial overgrowth had an overwhelmingly higher frequency of arteries affected by coronary artery disease [30]. In murine research, investigators found that by rebalancing the species in the gut microbiome, they were able to reduce cholesterols levels and dramatically slow the buildup of fatty deposits in arteries [29]. Another potential mechanism involves formation of immunogens (advanced glycation end-products (enFruAGE)) in the gut, via glycation, between between unabsorbed/luminal fructose and partially digested dietary proteins [67] that once absorbed contribute to atherosclerotic plaque [10]. Advanced glycation end-products (AGEs) and their receptors may play important roles in the development and progression of CHD [68]. Recent biochemical [69-72] and other findings [73] are consistent with the possibility that AGEs form in the gut. Notably, n-carboxymethyl lysine (CML)-a well studied AGEformed under conditions consistent with the intestines, well within the time window of digestion, but not with glucose [69-71]. Unabsorbed fructose's high reactivity lies in the fact that it is in open chain form 400 times more than glucose [74].

It is increasingly evident that factors which disrupt the gut have far reaching consequences. The intestine's capacity to absorb free fructose is saturable and ranges widely from $\sim 5 \mathrm{~g}$ to $>50 \mathrm{~g}$ in healthy adults [13]. There are no known genetic factors associated with fructose malabsorption. Therefore, it is likely that fructose malabsorption is a man made problem, as most natural foods contain $\mathrm{a} \sim 1: 1$ fructose-to-glucose ratio [11]. It is noteworthy that epidemiological studies show that even moderate intake of high excess free fructose beverages (HFCS sweetened soda, fruit drinks, and apple juice) increases the risk $[75,76]$ and prevalence of asthma [77-86], and young adult idiopathic arthris [87] - CHD comorbidities $[88,89]$. There were no associations with orange juice [75-77, 79, 87]. The black/white CHD death disparity that began during the 1980's corresponds with the black/white childhood asthma disparity [90-92] that inexplicably grew twofold from the 1980's to 2010.

This study has limitations and may not be generalizable to other population settings, as the JHS is specific to African Americans. First, the exclusions (7.5\%) due to preexisting CHD, likely reduced statistical power with the effect of widening the $95 \%$ confidence intervals. Second, outcomes were based on a combination of data that included self-reports, which are subject to reporting bias. However, associations between HFCS sweetened beverages and $\mathrm{CHD}$ are consistent with existing literature $[6,7$, 10]. Third, we may be underestimating the true $\mathrm{CHD}$ risk from HFCS intake, as HFCS is not exclusive to beverages. Although two-thirds of all HFCS consumed in the United States is in beverages [93, 94], HFCS is ubiquitous in the US food supply and is widely used to sweeten processed foods, including cookies, ice cream, ketchup, crackers, bread, soups, cereals, spaghetti sauce, desserts, and others $[32,33]$. Fourth, this study does not account for additions to daily excess-free-fructose intake from non-HFCS sources, including $100 \%$ apple juice, a juice with $a \geq 2: 1$ fructose-to-glucose ratio [11], agave syrup ( $\geq 60$ fructose) [55], crystalline fructose, and fruits that have high fructose-to-glucose ratios including, apples, pears, watermelon, and mangoes [11]. Therefore, the true CHD risk from daily excess-free-fructose intakes may be understated. Fifth, possible changes in food intake frequencies throughout the JHS study period were not accounted for, as the JHS collected intake frequencies once, during exam 1. This may have introduced a margin of error into our results. However, notwithstanding magnitude of association differences, these results are consistent with existing studies of SSB and CHD $[6,7,10]$, and excess-free-fructose and CHD [10].

\section{Conclusion}

The ubiquitous presence of HFCS in the US food supply over the past 30 years may have exposed African Americans to increased CHD risk, as they are more likely to be fructose malabsorbers and thereby suffer from unabsorbed fructose induced dysbiosis and gut formation of atherosclerotic advanced glycation end-products linked to CHD.

\section{Abbreviations}

AGE: Advanced glycation end-products; BMI: Body mass index; BP: Blood pressure; CDC: United States Centers for Disease Control; CEL: n-carboxyethyl lysine, a well studied advanced glycation end-product; CHD: Coronary heart disease; Cl: Confidence interval; CML: n-carboxymethyl lysine, a well studied advanced glycation end-product; CVD: Cardiovascular disease; EFF: Excessfree-fructose; enFruAGE: Extracellular newly-identified fructose associated advanced glycation end-products; FFQ: Food frequency questionnaire; GLUT5: Intestinal transporter of fructose when consumed in excess of glucose; GRAS: Generally recognized as safe; HDL: High density lipoprotein; HbA1c: Glycated hemoglobin; HFCS: High fructose corn syrup; HR: Hazard ratio; JHS: the Jackson Heart Study; LDL-c: Low density lipoprotein; NHAN ES: (US) National Health and Nutrition Examination Survey; NIAID: National Institute on Allergy and Infectious Diseases; RAGE: Receptors of advanced glycation end-products; SES: Socio-economic status; SSB: Sugar sweetened beverages; ttlEFF: Any combination of beverages high in excess-free-fructose including high fructose corn syrup sweetened soda, fruit drinks, and apple juice; US: United States

\section{Acknowledgements}

The views expressed in this manuscript are those of the authors and do not necessarily represent the views of the National Heart, Lung, and Blood Institute; the National Institutes of Health; or the U.S. Department of Health and Human Services. 


\section{Authors' contributions}

LRDC researched and developed the biochemical hypothesis, designed the epidemiology research, performed all of the analyses and wrote the manuscript. LRDC holds a Master's of Science in Biochemistry, Molecular Biology, New York Medical College, Valhalla, NY; she has additionally completed advanced coursework in immunology, epidemiology, and biostatistics. BJA provided critical review and helped edit the manuscript. KLT provided critical review, reviewed all statistical analyses and contributed to editing the manuscript. All authors read and approved the final manuscript

\section{Funding}

This analysis was not supported with external funding. The Jackson Heart Study (JHS) is supported and conducted in collaboration with Jackson State University (HHSN268201300049C and HHSN268201300050C), Tougaloo College (HHSN268201300048C), and the University of Mississippi Medical Center (HHSN268201300046C and HHSN268201300047C) contracts from the National Heart, Lung, and Blood Institute (NHLBI) and the National Institute for Minority Health and Health Disparities (NIMHD). This analysis was not supported with external funding.

\section{Availability of data and materials}

Access to data for this analysis was provided by the Jackson Heart Study upon approval of our proposal by the Proposal and Presentation Committee and after signing the Jackson Heart Study data use agreement, Publication ID: P0687.

\section{Ethics approval and consent to participate}

The Jackson Heart Study was approved by the US National Heart, Lung and Blood Institute of the National Institutes of Health, and all participants gave written consent. This project was approved for "exempt status" by the University of Massachusetts Lowell Institutional Review Board.

\section{Consent for publication}

Not applicable.

\section{Competing interests}

All authors declare no conflict of interest.

\section{Author details}

${ }^{1}$ Independent Researcher, M.Sc. Biochemistry, Molecular Biology, P.O. Box 5542, Eugene, OR 97405, USA. ${ }^{2}$ Department of Primary Care, Virginia Mason Medical Center, Seattle, WA, USA. ${ }^{3}$ Department of Biomedical and Nutritional Sciences, University of Massachusetts Lowell, Lowell, MA, USA.

Received: 12 August 2020 Accepted: 31 October 2020

Published online: 08 December 2020

\section{References}

1. Kramer MR, Valderrama AL, Casper ML. Decomposing Black-White disparities in heart disease mortality in the United States, 1973-2010: an age-periodcohort analysis. Am J Epidemiol. 2015;182(4):302-12. https://doi.org/10.1093/ aje/kwv050 https://www.ncbi.nlm.nih.gov/pubmed/26199382.

2. United States Department of Health and Human Services Office of Minority Health. Heart Disease and African Americans. https://minorityhealth.hhs.gov/ omh/browse.aspx?lvl=4\&lvlid=19 Accessed 22 Dec 2017.

3. Yusuf S, Reddy S, Ounpuu S, Anand S. Global burden of cardiovascular diseases: part I: general considerations, the epidemiologic transition, risk factors, and impact of urbanization. Circulation. 2001;104(22):2746-53 Review. PubMed PMID: 11723030.

4. Davis AM, Vinci LM, Okwuosa TM, Chase AR, Huang ES. Cardiovascular health disparities: a systematic review of health care interventions. Med Care Res Rev. 2007;64(5 Suppl):29S-100S. https://doi.org/10.1177/ 1077558707305416.

5. Kirby JB, Kaneda T. Unhealthy and uninsured: exploring racial differences in health and health insurance coverage using a life table approach. Demography. 2010;47(4):1035-51. https://doi.org/10.1007/BF03213738.

6. de Koning L, Malik VS, Kellogg MD, Rimm EB, Willett WC, Hu FB. Sweetened beverage consumption, incident coronary heart disease, and biomarkers of risk in men. Circulation. 2012;125(14):1735-41, S1. https://doi.org/10.1161/ CIRCULATIONAHA.111.067017 Epub 2012 Mar 12. PubMed PMID: 22412070;
PubMed Central PMCID: PMC3368965. https://www.ncbi.nlm.nih.gov/ pubmed/22412070.

7. Fung TT, Malik V, Rexrode KM, Manson JE, Willett WC, Hu FB. Sweetened beverage consumption and risk of coronary heart disease in women. Am J Clin Nutr. 2009:89(4):1037-42. https://doi.org/10.3945/ajcn.2008.27140 https://www.ncbi.nlm.nih.gov/pubmed/19211821.

8. Malik VS, Hu FB. Fructose and Cardiometabolic Health: What the Evidence From Sugar-Sweetened Beverages Tells Us. J Am Coll Cardiol 2015;66(14): 1615-1624. doi: https://doi.org/10.1016/j.jacc.2015.08.025. Review. PubMed PMID: 26429086; PubMed Central PMCID: PMC4592517.

9. Han E, Powell LM. Consumption patterns of sugar-sweetened beverages in the United States. J Acad Nutr Diet. 2013;113(1):43-53. https://doi.org/10. 1016/j.jand.2012.09.016 PubMed PMID: 23260723; PubMed Central PMCID: PMC3662243.

10. DeChristopher LR, Uribarri J, Tucker KL. Intake of high-fructose corn syrup sweetened soft drinks, fruit drinks and apple juice is associated with prevalent coronary heart disease in US adults, aged 20-30 years. Biomed Central Nutrition Journal. 2017;3:51. https://doi.org/10.1186/s40795-0170168-9.

11. U.S. Department of Agriculture, Agricultural Research Service. USDA National Nutrient Database for Standard Reference, Release 28. Nutrient Data Laboratory Home Page, https://www.ars.usda.gov/ARSUserFiles/80400525/ Data/SR/SR28/reports/sr28fg09.pdf Accessed 23 Aug 2017.

12. Atkinson FS, Foster-Powell K, Brand-Miller JC. International tables of glycemic index and glycemic load values: 2008. Diabetes Care. 2008;31(12): 2281-3. https://doi.org/10.2337/dc08-1239 http://care.diabetesjournals.org/ content/31/12/2281.

13. Hannou SA, Haslam DE, McKeown NM, Herman MA. Fructose metabolism and metabolic disease. J Clin Invest. 2018;128(2):545-55. https://doi.org/10. 1172/JCl96702 Epub 2018 Feb 1. Review. PubMed PMID: 29388924; PubMed Central PMCID: PMC5785258.

14. Jones HF, Burt E, Dowling K, Davidson G, Brooks DA, Butler RN. Effect of age on fructose malabsorption in children presenting with gastrointestinal symptoms. J Pediatr Gastroenterol Nutr. 2011;52(5):581-4. https://doi.org/10. 1097/MPG.0b013e3181fd1315 PubMed PMID: 21502829. https://www.ncbi. nlm.nih.gov/pubmed/21502829.

15. Smith MM, Davis M, Chasalow Fl, Lifshitz F. Carbohydrate absorption from fruit juice in young children. Pediatrics. 1995;95(3):340-344. PubMed PMID: 7862470. https://www.ncbi.nlm.nih.gov/pubmed/7862470.

16. Riby JE, Fujisawa T, Kretchmer N. Fructose absorption. Am J Clin Nutr 1993; 58(5 Suppl):748S-753S. Review. PubMed PMID: 8213606.

17. Ebert K, Witt H. Fructose malabsorption. Molecular and Cellular Pediatrics. 2016;3:10. doi:https://doi.org/10.1186/s40348-016-0035-9. PubMed PMCID: PMC4755956. https://www.ncbi.nlm.nih.gov/pubmed/26883354.

18. Hoekstra JH, van Kempen AA, Bijl SB, Kneepkens CM. Fructose breath hydrogen tests. Arch Dis Child 1993;68(1):136-138. PubMed PMID: 8435000; PubMed Central PMCID: PMC1029203. http://adc.bmj.com/content/ archdischild/68/1/136.full.pdf.

19. Biesiekierski JR. Fructose-induced symptoms beyond malabsorption in FGID. United European Gastroenterol J. 2014;2(1):10-3. https://doi.org/10.1177/ 2050640613510905 https://www.ncbi.nlm.nih.gov/pmc/articles/PMC4040804/.

20. Gibson PR, Newnham E, Barrett JS, Shepherd SJ, Muir JG. Review article: fructose malabsorption and the bigger picture. Aliment Pharmacol Ther. 2007;25:349-63 https://www.ncbi.nlm.nih.gov/pubmed/17217453.

21. Rumessen JJ. Fructose and related food carbohydrates. Sources, intake, absorption, and clinical implications. Scand J Gastroenterol. 1992;27:819-28 https://www.ncbi.nlm.nih.gov/pubmed/1439534.

22. Beyer PL, Caviar EM, McCallum RW. Fructose intake at current levels in the United States may cause gastrointestinal distress in normal adults. J Am Diet Assoc. 2005;105:1559-66 https://www.ncbi.nlm.nih.gov/pubmed/16183355.

23. U.S. Department of Agriculture, Economic Research Service. 2012. The ERS food availability (per capita) data system. Economic Research Service Home Page, http://www.ers.usda.gov/data-products/food-availability-(per-capita)data-system.aspx\#.

24. U.S. Department of Agriculture, Economic Research Service. Sugars and Sweeteners, Background, High Fructose Corn Syrup Production and Prices. http://www.ers.usda.gov/topics/crops/sugar-sweeteners/background/ Accessed 3/15/2017.

25. Strom S. U.S. Cuts Estimate of Sugar Intake. The New York Times.2012. http://www.nytimes.com/2012/10/27/business/us-cuts-estimate-of-sugarintake-of-typical-american.html. Accessed 22 Dec 2017. 
26. Haley S. Sugars and Sweeteners Outlook. United States Department of Agriculture. SSS-M-270. 2011. 6-9. http://usda.mannlib.cornell.edu/usda/ers/ SSS//2010s/2011/SSS-02-14-2011.pdf. Accessed 22 Dec 2017.

27. Haley S. Sugars and Sweeteners Outlook. United States Department of Agriculture. SSM-M-286; 2012. p. 16-9. http://usda.mannlib.cornell.edu/usda/ ers/SSS//2010s/2012/SSS-06-18-2012.pdf. Accessed 22 Dec 2017.

28. Ferraris RP, Choe JY, Patel CR. Intestinal absorption of fructose. Annu Rev Nutr. 2018;38:41-67. https://doi.org/10.1146/annurev-nutr-082117-051707 https://pubmed.ncbi.nlm.nih.gov/29751733/.

29. Chen PB, Black AS, Sobel AL, et al. Directed remodeling of the mouse gut microbiome inhibits the development of atherosclerosis [published online ahead of print, 2020 Jun 15]. Nat Biotechnol. 2020. https://doi.org/10.1038/ s41587-020-0549-5.

30. Shen B. Cleveland Clinic. Consult QD. Researchers Discover Gut-Heart Connection in Coronary Artery Disease https://consultad.clevelandclinic.org/ researchers-discover-gut-heart-connection-in-coronary-artery-disease/. Accessed 10 Nov 2020

31. Walker RW, Lê KA, Davis J, Alderete TL, Cherry R, Lebel S, Goran MI. High rates of fructose malabsorption are associated with reduced liver fat in obese African Americans. J Am Coll Nutr. 2012;31(5):369-74 PubMed PMID 23529994. http://www.goranlab.com/pdf/369.full.pdf.

32. Morrison RM, Buzby JC. Guess Who's Turning 100? Tracking a Century of American Eating. United States Department of Agriculture, Economic Research Service. Amber Waves. 2010; https://www.ers.usda.gov/amberwaves/2010/march/guess-who-s-turning-100tracking-a-century-of-americaneating/. Accessed 22 Dec 2017

33. Wells HF, Buzby JC. High-Fructose Corn Syrup Usage May Be Leveling Off. United States Department of Agriculture. Econ Res Serv. 2008; https://www. ers.usda.gov/amber-waves/2008/february/high-fructose-corn-syrup-usagemay-be-leveling-off/. Accessed 22 Dec 2017.

34. U.S. Food and Drug Administration. Food Additives and Petitions. High Fructose Corn Syrup Questions and Answers. https://www.fda.gov/food/ food-additives-petitions/high-fructose-corn-syrup-questions-and-answers. Accessed 10 Nov 2020.

35. Ventura EE, Davis JN, Goran MI. Sugar content of popular sweetened beverages based on objective laboratory analysis: focus on fructose content. Obesity (Silver Spring). 2011;19(4):868-74. https://doi.org/10.1038/oby.2010. 255 Epub 2010 Oct 14. PubMed PMID: 20948525. https://www.ncbi.nlm.nih. gov/pubmed/20948525.

36. Walker RW, Dumke KA, Goran MI. Fructose content in popular beverages made with and without high-fructose corn syrup. Nutrition. 2014;30(7-8): 928-935. doi: https://doi.org/10.1016/j.nut.2014.04.003. Epub 2014 Apr 18. PubMed PMID: 24985013. https://www.sciencedirect.com/science/article/pii/ S0899900714001920.

37. Federal Register Volume 61, Number 165. August 23, 1996. Rules and Regulations, pages 43447-43450. From the Federal Register Online via the Government Publishing Office [FR Doc No.: 9621482]. https://www.gpo.gov/ fdsys/pkg/FR-1996-08-23/html/96-21482.htm\%20 Accessed 23 Nov 2019.

38. United States National Heart, Lung, and Blood Institute (NHLBI) and National Institute on Minority Health and Health Disparities (NIMHD). Jackson Heart Study (JHS) of Cardiovascular Diseases in African Americans (JHS). Study record. ClinicalTrials.gov Identifier: NCT00005485. https://clinicaltrials.gov/ ct2/show/NCT00005485 and https://www.jacksonheartstudy.org/. Accessed 10 Nov 2020

39. Duffey KJ, Popkin BM. High-fructose corn syrup: is this what's for dinner? Am J Clin Nutr 2008;88(6):1722S-1732S. doi: https://doi.org/10.3945/ajcn. 2008.25825C. PubMed PMID: 19064537; PubMed Central PMCID: PMC2746720.

40. Ogden, C.L., Ph.D., M.R.P.; Kit, B.R.,M.D., M.P.H.; Carroll, M. D, M.S.P.H.; and Park, S., Ph.D., M.S. Consumption of Sugar Drinks in the United States, 2005 2008. NCHS Data Brief, Number 71, August 2011. http://www.cdc.gov/nchs/ data/databriefs/db71.htm. Accessed 10 Nov 2020.

41. Vartanian LR, Schwartz MB, Brownell KD. Effects of soft drink consumption on nutrition and health: a systematic review and meta-analysis. Am J Public Health. 2007:97(4):667-75 Epub 2007 Feb 28. Review. PubMed PMID: 17329656; PubMed Central PMCID: PMC1829363. https://www.ncbi.nlm.nih. gov/pmc/articles/PMC1829363/.

42. Tucker KL, Maras J, Champagne C, Connell C, Goolsby S, Weber J, Zaghloul S, Carithers T, Bogle ML. A regional food-frequency questionnaire for the US Mississippi Delta. Public Health Nutr. 2005;8(1):87-96 PubMed PMID: 15705249.
43. Subar AF, Dodd KW, Guenther PM, Kipnis V, Midthune D, McDowell M, Tooze JA, Freedman LS, Krebs-Smith SM. The food propensity questionnaire: concept, development, and validation for use as a covariate in a model to estimate usual food intake. J Am Diet Assoc 2006;106(10):1556-1563. PubMed PMID: 17000188. https://www.ncbi.nlm.nih.gov/pubmed/19559135. 44. Carithers TC, Talegawkar SA, Rowser ML, et al. Validity and calibration of food frequency questionnaires used with African American adults in the Jackson heart study. J Am Diet Assoc. 2009;109(7):1184-93. https://doi.org/ 10.1016/j.jada.2009.04.005.

45. Keku E, Rosamond W, Taylor HA Jr, Garrison R, Wyatt SB, Richard M, Jenkins B, Reeves L, Sarpong D. Cardiovascular disease event classification in the Jackson Heart Study: methods and procedures. Ethn Dis. 2005;15(4 Suppl 6): S6-62-70 PubMed PMID: 16317987.

46. Guenther PM, Kirkpatrick SI, Reedy J, Krebs-Smith SM, Buckman DW, Dodd KW, Casavale KO, Carroll RJ. The Healthy Eating Index-2010 is a valid and reliable measure of diet quality according to the 2010 Dietary Guidelines for Americans. J Nutr 2014;144(3):399-407. doi: https://doi.org/10.3945/jn.113. 183079. Epub 2014 Jan 22. PubMed PMID: 24453128; PubMed Central PMCI D: PMC3927552.

47. Wilson MM, Reedy J, Krebs-Smith SM. American Diet Quality: Where It Is, Where It Is Heading, and What It Could Be. J Acad Nutr Diet. 2015;116(2): 302-10.e1 https://www.ncbi.nlm.nih.gov/pmc/articles/PMC4733413/.

48. Joshi PH, Khokhar AA, Massaro JM, Lirette ST, Griswold ME, Martin SS, Blaha MJ, Kulkarni KR, Correa A, D'Agostino RB Sr, Jones SR, Toth PP. Lipoprotein Investigators Collaborative (LIC) Study Group.. Remnant Lipoprotein Cholesterol and Incident Coronary Heart Disease: The Jackson Heart and Framingham Offspring Cohort Studies. J Am Heart Assoc. 2016;5(5). https:// doi.org/10.1161/JAHA.115.002765 PubMed PMID: 27130348; PubMed Central PMCID: PMC4889167.

49. Stanhope $K L$, Havel PJ. Fructose consumption: potential mechanisms for its effects to increase visceral adiposity and induce dyslipidemia and insulin resistance. Curr Opin Lipidol. 2008;19(1):16-24. https://doi.org/10.1097/MOL. Ob013e3282f2b24a.

50. Hieronimus B, Griffen SC, Keim NL, et al. Effects of Fructose or Glucose on Circulating ApoCIII and Triglyceride and Cholesterol Content of Lipoprotein Subfractions in Humans. J Clin Med. 2019;8(7):913. Published 2019 Jun 26. https://doi.org/10.3390/jcm8070913.

51. Bacha F, Saad R, Gungor N, Janosky J, Arslanian SA. Obesity, regional fat distribution, and syndrome $X$ in obese black versus white adolescents: race differential in diabetogenic and atherogenic risk factors. J Clin Endocrinol Metab. 2003:88(6):2534-40 PubMed PMID: 12788850.

52. Kearns, C. E., L. A. Schmidt, and S. A. Glantz. 2016 Sugar industry and coronary heart disease research. An internal analysis of internal industry documents JAMA Internal Medicine (online) doi: https://doi.org/10.1001/ jamainternmed.2016.5394. https://www.ncbi.nlm.nih.gov/pubmed/27617709.

53. Fox A. Complaint for damages and injunctive relief for false advertising in the violation of (1) the Lantham Act (15 U.S.C. \& 1125(a)), and (2) California's Unfair Competition Law (Cal. Bus. \& Prof. Code \& 17200, Et. seq. Filed 4/22/ 2011. https://www.scribd.com/document/288646633/Western-SugarCooperative-et-al-v-Archer-Daniels-Midland-Company-et-al-Complaint. Accessed 10 Nov 2020.

54. Fox A. Second amended complaint for damages and injunctive relief for false advertising in violation of the Lantham Act. Filed 11/18/2011. https:// www.scribd.com/document/94868291/2d-Am-Complaint-Western-SugarCoop-v-Archer-Daniels-Midland. Accessed 10 Nov 2020.

55. Mellado-Mojica E, López MG. Identification, classification, and discrimination of agave syrups from natural sweeteners by infrared spectroscopy and HPAEC-PAD. Food Chem. 2015;167:349-57. https://doi.org/10.1016/j. foodchem.2014.06.111 Epub 2014 Jul 9. PubMed PMID: 25148997. https:// www.ncbi.nlm.nih.gov/pubmed/25148997.

56. White JS, Hobbs LJ, Fernandez S. Fructose content and composition of commercial HFCS-sweetened carbonated beverages. Int J Obes. 2015;39(1): 176-82. https://doi.org/10.1038/ijo.2014.73 Epub 2014 May 6. Review. PubMed PMID: 24798032; PubMed Central PMCID: PMC4285619.

57. Tuck CJ, Ross LA, Gibson PR, Barrett JS, Muir JG. Adding glucose to food and solutions to enhance fructose absorption is not effective in preventing fructose-induced functional gastrointestinal symptoms: randomised controlled trials in patients with fructose malabsorption. J Hum Nutr Diet 2017;30(1):73-82. doi: https://doi.org/10.1111/jhn.12409. Epub 2016 Sep 7. PubMed PMID: 27600184. https://www.ncbi.nlm.nih. gov/pubmed/27600184. 
58. Röder PV, Geillinger KE, Zietek TS, Thorens B, Koepsell H, Daniel H. The role of SGLT1 and GLUT2 in intestinal glucose transport and sensing. Plos One. 2014;9(2):e89977. https://doi.org/10.1371/journal.pone.0089977 eCollection 2014. PubMed PMID: 24587162; PubMed Central PMCID: PMC3935955.

59. Helliwell PA, Richardson M, Affleck J, Kellett GL. Stimulation of fructose transport across the intestinal brush-border membrane by PMA is mediated by GLUT2 and dynamically regulated by protein kinase C. Biochem J. 2000; 350(Pt. 1):149-54.

60. Kellett GL, Brot-Laroche E. Apical GLUT2: a major pathway of intestinal sugar absorption. Diabetes. 2005;54:3056-62.

61. Helliwell PA, Richardson M, Affleck J, Kellett GL. Regulation of GLUT5, GLUT2 and intestinal brush-border fructose absorption by the extracellular signalregulated kinase, p38 mitogen-activated kinase and phosphatidylinositol 3kinase intracellular signalling pathways: implications for adaptation to diabetes. Biochem J. 2000;350(Pt 1):163-9.

62. Patel C, Douard V, Yu S, Gao N, Ferraris RP. Transport, metabolism, and endosomal trafficking-dependent regulation of intestinal fructose absorption. FASEB J 2015;29(9):4046-4058. doi: https://doi.org/10.1096/fj.15272195. Epub 2015 Jun 12. PubMed PMID: 26071406; PubMed Central PMCI D: PMC4550372

63. Eshak ES, Iso H, Kokubo Y, Saito I, Yamagishi K, Inoue M, Tsugane S. Soft drink intake in relation to incident ischemic heart disease, stroke, and stroke subtypes in Japanese men and women: the Japan Public Health Centrebased study cohort I. Am J Clin Nutr. 2012;96(6):1390-7. https://doi.org/10. 3945/ajcn.112.037903 Epub 2012 Oct 17. PubMed PMID: 23076619.

64. Market Evaluation Consumption and Statistics Committee. International Sugar Organization. Alternative Sweeteners in a High Sugar Price Environment. 2012. https://www.scribd.com/document/245129336/MECAS-12-04-AlternativeSweeteners-in-a-Higher-Sugar-Price-Environment-English.

65. Yang Q, Zhang Z, Gregg EW, Flanders WD, Merritt R, Hu FB. Added sugar intake and cardiovascular diseases mortality among US adults. JAMA Intern Med. 2014;174(4):516-24. https://doi.org/10.1001/jamainternmed.2013.13563 PubMed PMID: 24493081.

66. Hieronimus B, Medici V, Bremer AA, Lee V, Nunez MV, Sigala DM, Keim NL, Havel PJ, Stanhope KL. Synergistic effects of fructose and glucose on lipoprotein risk factors for cardiovascular disease in young adults. Metabolism. 2020;112:154356. https://doi.org/10.1016/j.metabol.2020.154356 [Epub ahead of print] PubMed PMID: 32916151.

67. DeChristopher, Luanne Robalo. Consumption of Fructose and High Fructose Corn Syrup: Is Fructositis triggered bronchitis, asthma, \& auto-immune reactivity merely a side bar in the Etiology of Metabolic Syndrome II (to be defined)? - Evidence and a Hypothesis. New York Medical College Library, 2012, print. Online at https://www.researchgate.net/publication/276920662_ Consumption_of_Fructose_and_High_Fructose_Corn_Syrup_Is_Fructositis_ triggered_bronchitis_asthma_auto-immune_reactivity_merely_a_side_bar_ in_the_Etiology_of_Metabolic_Syndrome_II_to_be_defined_-_Eviden. Accessed 10 Nov 2020

68. Fishman SL, Sonmez H, Basman C, et al. The role of advanced glycation end-products in the development of coronary artery disease in patients with and without diabetes mellitus: a review. Mol Med. 2018;24:59. https:// doi.org/10.1186/s10020-018-0060-3.

69. Bains Y, Gugliucci A, Caccavello R. Advanced glycation endproducts form during ovalbumin digestion in the presence of fructose: Inhibition by chlorogenic acid. Fitoterapia; 2017. https://doi.org/10.1016/j.fitote.2017.05.003.

70. Bains Y, Gugliucci A. Ilex paraguariensis and its main component chlorogenic acid inhibit fructose formation of advanced glycation endproducts with amino acids at conditions compatible with those in the digestive system. Fitoterapia. 2016;117:6-10. https://doi.org/10.1016/j.fitote. 2016.12.006 [Epub ahead of print] PubMed PMID: 28012919. https://www. sciencedirect.com/science/article/pii/S0367326X16308619.

71. Martinez-Saez N, Fernandez-Gomez B, Cai W, Uribarri J, Dolores del Castillo M. In vitro formation of Maillard reaction products during simulated digestion of meal-resembling systems. In Food Research International. 2017. ISSN 0963-9969, https://doi.org/10.1016/.jfoodres.2017.09.056. (http://www. sciencedirect.com/science/article/pii/S0963996917306415).

72. van der Lugt T, Venema K, van Leeuwen S, Vrolijk MF, Opperhuizen A, Bast A. Gastrointestinal digestion of dietary advanced glycation endproducts using an in vitro model of the gastrointestinal tract (TIM-1). Food Funct. 2020;11(7):6297-307. https://doi.org/10.1039/d0fo00450b.

73. DeChristopher LR. Perspective: The Paradox in Dietary Advanced Glycation End Products Research-The Source of the Serum and Urinary Advanced
Glycation End Products Is the Intestines, Not the Food. Adv Nutr. 2017;8(5): 679-83. https://doi.org/10.3945/an.117.016154 Print 2017 Sep. PubMed PMID: 28916568; PubMed Central PMCID: PMC5593110.

74. Clemens RA, Jones JM, Kern M, Lee SY, Mayhew EJ, Slavin JL, Zivanovic S. Functionality of Sugars in Foods and Health. Comprehensive Reviews in Food Science and Food Safety; 2016. https://doi.org/10.1111/1541-4337. 12194.

75. DeChristopher $L R$, Tucker KL. Excess free fructose, apple juice, high fructose corn syrup and childhood asthma risk - the National Children's Study. Nutr J. 2020;19(1):60. Published 2020 Jun 23. https://doi.org/10.1186/s12937-02000578-0.

76. DeChristopher LR, Tucker KL. Excess-free-fructose, high-fructose corn syrup and adult asthma: the Framingham Offspring Cohort. Br J Nutr. 2018:1-11. https://doi.org/10.1017/S0007114518000417 [Epub ahead of print] PubMed PMID: 29587887. https://www.ncbi.nlm.nih.gov/pubmed/29587887.

77. DeChristopher LR, Uribarri J, Tucker KL. Intakes of apple juice, fruit drinks and soda are associated with prevalent asthma in US children aged 2-9 years. Public Health Nutr. 2015:1-8 [Epub ahead of print] PubMed PMID: 25857343. https://www.ncbi.n/m.nih.gov/pubmed/25857343. Accessed 10 Nov 2020.

78. DeChristopher $L R$, Uribarri J, Tucker $K \mathrm{~L}$. The link between soda intake and asthma: science points to the high-fructose corn syrup, not the preservatives: a commentary. Nutr Diabetes. 2016;6(11):e234. https://doi.org/ 10.1038/nutd.2016.46 PubMed PMID: 27892935; PubMed Central PMCID: PMC5133361. https://www.ncbi.nlm.nih.gov/pubmed/27892935.

79. Wright LS, Rifas-Shiman S, Oken E, Gillman M, Litonjua AA, Gold DR. Maternal Prenatal Intake of Fructose Is Associated with Asthma in Children. J Allergy Clin Immunol. 135(2):AB228. https://doi.org/10.1016/j.jaci.2014.12. 1678.

80. Wright LS, Rifas-Shiman SL, Oken E, Litonjua AA, Gold DR. Prenatal and Early-Life Fructose, Fructose-Containing Beverages, and Mid-Childhood Asthma. Ann Am Thorac Soc. 2017. https://doi.org/10.1513/AnnalsATS. 201707-5300C [Epub ahead of print] PubMed PMID: 29219619.

81. Yu R, Yang B, Cai L, Lu X, Wang X. Excess Free Fructose Beverages and Allergy in Children and Adolescents: Results From NHANES 2005-2006. Ann Fam Med. 2018;16(5):408-18. https://doi.org/10.1370/afm.2292 PubMed PMID: 30201637

82. Park S, Blanck HM, Sherry B, Jones SE, et al. Regular-soda intake independent of weight status is associated with asthma among US high school students. J Acad Nutr Diet 2013;113(1):106-111. doi: https://doi.org/ 10.1016/j.jand.2012.09.020. PubMed PMID: 23260727; PubMed Central PMCI D: PMC4470486.

83. Park S, Akinbami LJ, McGuire LC, Blanck HM. Association of sugarsweetened beverage intake frequency and asthma among U.S. adults, 2013. Prev Med. 2016;91:58-61. https://doi.org/10.1016/j.ypmed.2016.08.004 [Epub ahead of print]; PubMed PMID: 27496394.

84. Cisneros R, Gonzalez M, Brown P, Schweizer D. Soda consumption and hospita admissions among Californian adults with asthma. J Asthma. 2017;54(4):371-5. https://doi.org/10.1080/02770903.2016.1218014. Epub 2016 Aug 5.

85. DeChristopher LR. Excess free fructose and childhood asthma. Eur J Clin Nutr. 2015 Jun 17. doi: https://doi.org/10.1038/ejcn.2015.101. [Epub ahead of print] PubMed PMID: 26081487. https://www.ncbi.nlm.nih.gov/pubmed/26 081487 https://www.researchgate.net/publication/278794143_Excess_free_ fructose_and_childhood_asthma.

86. Berentzen NE, van Stokkom VL, Gehring U, Koppelman GH, Schaap LA, Smit $\mathrm{HA}$, et al. Associations of sugar-containing beverages with asthma prevalence in 11-year-old children: the PIAMA birth cohort. Eur J Clin Nutr. 2015;69:303-8. https://doi.org/10.1038/ejcn.2014.153 PubMed PMID: 25117998.

87. DeChristopher LR, Uribarri J, Tucker KL. Intake of high-fructose corn syrup sweetened soft drinks, fruit drinks and apple juice is associated with prevalent arthritis in US adults, aged 20-30 years. Nutr Diab. 2016;6(3):e199. Published 2016 Mar 7. https://doi.org/10.1038/nutd.2016.7.

88. Yun HD, Knoebel E, Fenta Y, Gabriel SE, Leibson CL, Loftus EV Jr, et al. Asthma and proinflammatory conditions: a population-based retrospective matched cohort study. Mayo Clin Proc. 2012;87(10):953-60. https:/doi.org/ 10.1016/j.mayocp.2012.05.020 Epub 2012 Sep 12. PubMed PMID: 22980164; PubMed Central PMCID: PMC3538394.

89. Jagpal A, Navarro-Millán I. Cardiovascular co-morbidity in patients with rheumatoid arthritis: a narrative review of risk factors, cardiovascular risk assessment and treatment. BMC Rheumatol. 2018;2:10. https://doi.org/10. 1186/s41927-018-0014-y. 
90. Akinbami $\sqcup$, Simon AE, Rossen LM. Changing Trends in Asthma Prevalence Among Children. Pediatrics. 2016;137(1). https://doi.org/10.1542/peds.20152354 Epub 2015 Dec 28. PubMed PMID: 26712860; PubMed Central PMCID: PMC4755484.

91. National Public Radio. Health News. Childhood Asthma Rates Level Off, But Racial Disparities Remain. 2015. https://www.npr.org/sections/health-shots/2 015/12/28/460845335/childhood-asthma-rates-level-off-but-racial-disparitiesremain.

92. Akinbami L, Rossen LM, Fakhouri THI, Fryar CD. Asthma prevalence trends by weight status among US children aged 2-19 years, 1988-2014. Pediatr Obes. 2018;13(6):393-6. https://doi.org/10.1111/ijpo.12246 Epub 2017 Nov 12. PubMed PMID: 29130640.

93. Bray GA. Energy and fructose from beverages sweetened with sugar or high-fructose corn syrup pose a health risk for some people. Adv Nutr 2013; 4(2):220-225. doi: https://doi.org/10.3945/an.112.002816. Review. PubMed PMID: 23493538; PubMed Central PMCID: PMC3649102.

94. Bray GA, Nielsen SJ, Popkin BM. Consumption of high-fructose corn syrup in beverages may play a role in the epidemic of obesity. Am J Clin Nutr. 2004; 79(4):537-43 Review. Erratum in: Am J Clin Nutr. 2004 Oct;80(4):1090. PubMed PMID: 15051594.

\section{Publisher's Note}

Springer Nature remains neutral with regard to jurisdictional claims in published maps and institutional affiliations.

Ready to submit your research? Choose BMC and benefit from:

- fast, convenient online submission

- thorough peer review by experienced researchers in your field

- rapid publication on acceptance

- support for research data, including large and complex data types

- gold Open Access which fosters wider collaboration and increased citations

- maximum visibility for your research: over $100 \mathrm{M}$ website views per year

At $\mathrm{BMC}$, research is always in progress.

Learn more biomedcentral.com/submissions 